\title{
Functional Determinants for Radially Separable Partial Differential Operators
}

G. V. Dunne

Functional determinants of differential operators play a prominent role in many fields of theoretical and mathematical physics, ranging from condensed matter physics, to atomic, molecular and particle physics. They are, however, difficult to compute reliably in non-trivial cases. In one dimensional problems (i.e. functional determinants of ordinary differential operators), a classic result of Gel'fand and Yaglom greatly simplifies the computation of functional determinants. Here I report some recent progress in extending this approach to higher dimensions (i.e., functional determinants of partial differential operators), with applications in quantum field theory.

Keywords: quantum field theory, functional determinants, zeta functions, spectral theory, partial differential operators.

\section{Introduction and statement of results}

This paper considers the fundamental questions: what is the determinant of a partial differential operator, and how might one compute it?

Determinants of differential operators occur naturally in many applications in mathematical and theoretical physics, and also have inherent mathematical interest since they encode certain spectral properties of differential operators. Physically, such determinants arise, for example, in semiclassical approximations in quantum mechanics and quantum field theory, in grand canonical potentials in many-body theory and statistical mechanics, in gap equations in the mean-field approximation, in lattice gauge theory, and in gauge fixing (Faddeev-Popov determinant) for non-abelian gauge theory. Determinants of free Laplacians and free Dirac operators have been extensively studied [1-6], but much less is known about operators involving an arbitrary potential function. When the operator under consideration is an ordinary (i.e., one dimensional) differential operator, a beautiful general theory due to Gel'fand and Yaglom [7] has been developed for defining and computing the determinant [8-11]. In this paper I discuss attempts to extend these results to partial differential operators. Even for the simple radially separable case of the free Laplacian on a $2 d$ disc, the naive extension via a sum over partial waves of ordinary differential operators, leads to a divergence, as noted by Forman [9]. However, it turns out that this divergence has a clear physical meaning and can be understood in the context of renormalization in quantum field theory. This leads to finite, renormalized expressions [see Eqs. (6)-(8) below] for the determinant of such separable operators. The result for four dimensions was first found in [12] using radial WKB and an angular momentum cut-off regularization and renormalization [13], and then in [14] using the zeta function approach to determinants. The primary motivation of this work is for applications in quantum field theory, so we concentrate on examples in two, three and four dimensions, but the mathematical generalization to arbitrary dimension should be clear.

Consider the radially separable partial differential operators

$$
\mathcal{M}=-\Delta+V(r) ; \mathcal{M}^{\text {free }}=-\Delta
$$

where $\Delta$ is the Laplace operator in $\mid \mathrm{R}^{d}$, and $V(r)$ is a radial potential vanishing at infinity as $r^{-2-\varepsilon}$ for $d=2$ and $d=3$, and as $r^{-4-\varepsilon}$ for $d=4$. For $d=1$, with Dirichlet boundary conditions on the interval $[0, \infty)$, the results of Gel'fand and Yaglom [7] lead to the following simple expression for the determinant ratio:

$$
\frac{\operatorname{det}\left[\mathcal{M}+m^{2}\right]}{\operatorname{det}\left[\mathcal{M}^{\text {free }}+m^{2}\right]}=\frac{\psi(\infty)}{\psi^{\text {free }}(\infty)},
$$

where $\left[\mathcal{M}+m^{2}\right] \psi=0$, with initial value boundary conditions: $\psi(0)=0$ and $\psi^{\prime}(0)=1$. The function $\psi^{\text {free }}$ is defined similarly in terms of the free operator: $\left[\mathcal{M}^{\text {free }}+m^{2}\right]$. The squared mass, $m^{2}$, is important for physical applications, and plays the mathematical role of a spectral parameter. The result (4) is geometrically interesting, in addition to being computationally simple, as it means that the determinant is determined simply by the boundary values of the solutions of $\left[\mathcal{M}+m^{2}\right] \psi=0$, and no detailed information is needed concerning the actual spectrum of eigenvalues.

Now consider dimensions $d>1$. Since the potential is radial, $V=V(r)$, we can express the eigenfunctions of $\mathcal{M}$ as linear combinations of basis functions of the form

$$
\Psi(r, \vec{\theta})=\frac{1}{r^{(d-1) / 2}} \psi_{(l)}(r) Y_{(l)}(\vec{\theta}),
$$

where $Y_{(l)}(\vec{\theta})$ is a hyperspherical harmonic, labeled in part by a non-negative integer $l$, and the radial function $\psi_{(l)}(r)$ is an eigenfunction of the Schrödinger-like radial operator

$\mathcal{M}_{(l)} \equiv-\frac{d^{2}}{d r^{2}}+\frac{\left(l+\frac{d-3}{2}\right)\left(l+\frac{d-1}{2}\right)}{r^{2}}+V(r)$.

$\mathcal{M}_{(l)}^{\text {free }}$ is defined similarly, with the potential omitted: $V=0$. In dimension $d \geq 2$, the radial eigenfunctions $\psi_{(l)}$ have degeneracy given by

$$
\operatorname{deg}(l ; d) \equiv \frac{(2 l+d-2)(l+d-3) !}{l !(d-2) !} .
$$

Formally, for the separable operators in (1), the logarithm of the determinant ratio can be written as a sum over $l$ (weighted with the degeneracy factor) of the logarithm of one-dimensional determinant ratios,

$\ln \left(\frac{\operatorname{det}\left[\mathcal{M}+m^{2}\right]}{\operatorname{det}\left[\mathcal{M}^{\text {free }}+m^{2}\right]}\right)=\sum_{l=0}^{\infty} \operatorname{deg}(l ; d) \ln \left(\frac{\operatorname{det}\left[\mathcal{M}_{(l)}+m^{2}\right]}{\operatorname{det}\left[\mathcal{M}_{(l)}^{\text {free }}+m^{2}\right]}\right)$ 
Each term in the sum can be computed using the Sturm-Liouville extension [10] of the Gel'fand-Yaglom result (2). However, the $l$ sum in (5) is divergent, as noted by Forman [9] for the free Laplace operator in a two-dimensional disc. However, it is possible to understand this divergence and define a finite and renormalized determinant ratio for the radially separable partial differential operators (1). Specifically, we have found [1] the following simple expressions, which generalize (2) to higher dimensions:

$$
\begin{aligned}
& \left.\ln \left(\frac{\operatorname{det}\left[\mathcal{M}+m^{2}\right]}{\operatorname{det}\left[\mathcal{M}^{\text {free }}+m^{2}\right]}\right)\right|_{d=2}= \\
& \ln \left(\frac{\psi_{(0)}(\infty)}{\psi_{(0)}^{\text {free }}(\infty)}\right)+\sum_{l=1}^{\infty} 2\left\{\ln \left(\frac{\psi_{(l)}(\infty)}{\psi_{(l)}^{\text {free }}(\infty)}\right)-\frac{\int_{0}^{\infty} \mathrm{d} r r V(r)}{2 l}\right\} \\
& +\int_{0}^{\infty} \mathrm{d} r r V\left[\ln \left(\frac{\mu r}{2}\right)+\gamma\right] \\
& \left.\ln \left(\frac{\operatorname{det}\left[\mathcal{M}+m^{2}\right]}{\operatorname{det}\left[\mathcal{M}^{\text {free }}+m^{2}\right]}\right)\right|_{d=3}= \\
& \sum_{l=0}^{\infty}(2 l+1)\left\{\ln \left(\frac{\psi_{(l)}(\infty)}{\psi_{(l)}^{\text {free }}(\infty)}\right)-\frac{\int_{0}^{\infty} \mathrm{d} r r V(r)}{2\left(l+\frac{1}{2}\right)}\right\} \\
& \left.\ln \left(\frac{\operatorname{det}\left[\mathcal{M}+m^{2}\right]}{\operatorname{det}\left[\mathcal{M}^{\text {free }}+m^{2}\right]}\right)\right|_{d=4}= \\
& \sum_{l=0}^{\infty}(l+1)^{2}\left\{\ln \left(\frac{\psi_{(l)}(\infty)}{\psi_{(l)}^{\text {free }}(\infty)}\right)-\frac{\int_{0}^{\infty} \mathrm{d} r r V(r)}{2(l+1)}+\frac{\int_{0}^{\infty} \mathrm{d} r r^{3} V\left(V+2 m^{2}\right)}{8(l+1)^{3}}\right\}(8) \\
& -\frac{1}{8} \int_{0}^{\infty} \mathrm{d} r r^{3} V\left(V+2 m^{2}\right)\left[\ln \left(\frac{\mu r}{2}\right)+\gamma+1\right] .
\end{aligned}
$$

Here $\gamma$ is Euler's constant, and $\mu$ is a renormalization scale (defined in the next section), which is essential for physical applications, and which arises naturally in even dimensions. A conventional renormalization choice is to take $\mu=m$ in (6)-(8). In each of (6)-(8), the sum over $l$ is convergent once the indicated subtractions are made. The function $\psi_{(l)}(r)$ is the solution to the radial equation

$$
\begin{aligned}
{\left[\mathcal{M}_{(l)}+m^{2}\right] \psi_{(l)}(r) } & =0 \\
\psi_{(l)}(r) & \sim r^{l+\frac{(d-1)}{2}}, \quad \text { as } r \rightarrow 0 .
\end{aligned}
$$

The function $\psi_{(l)}^{\text {free }}(r)$ is defined similarly, with the same behavior as $r \rightarrow 0$, in terms of the operator $\left[\mathcal{M}_{(l)}^{\text {free }}+m^{2}\right]$. Thus, in $d$ dimensions, $\psi_{(l)}^{\text {free }}(r)$ is expressed as a Bessel function: $\psi_{(l)}^{\text {free }}(r)=\left(\frac{2}{m}\right)^{l+\frac{d}{2}-1} \Gamma\left(l+\frac{d}{2}\right) r^{\frac{1}{2}} I_{l+\frac{d}{2}-1}(m r)$.

Notice that the results (6)-(8) state once again that the determinant is determined by the boundary values of solutions of $\left[\mathcal{M}+m^{2}\right] \psi=0$, with the only additional information being a finite number of integrals involving the potential $V(r)$. We also stress the computational simplicity of (6)-(8), as the initial value problem (9) is trivial to implement numerically.

\section{Zeta function formalism}

The functional determinant can be defined in terms of a zeta function $[1,2,5]$ for the operator $\mathcal{M}$. For dimensional reasons, we define

$$
\zeta_{\frac{\left[\mathcal{M}+m^{2}\right]}{\mu^{2}}}(s)=\mu^{2 s} \zeta_{\left[\mathcal{M}+m^{2}\right]}(s)=\mu^{2 s} \sum_{\lambda}\left(\lambda+m^{2}\right)^{-s},
$$

where the sum is over the spectrum of $\mathcal{M}$, and $\mu$ is an arbitrary parameter with dimension of a mass. Physically, $\mu$ plays the role of a renormalization scale. Then the logarithm of the determinant is defined as $[1,2,5]$

$$
\begin{aligned}
\ln \operatorname{det}\left[\mathcal{M}+m^{2}\right] & \equiv-\zeta_{\frac{\left[\mathcal{M}+m^{2}\right]}{\mu^{2}}}^{\prime}(0) \\
& =-\ln \left(\mu^{2}\right) \zeta_{\left[\mathcal{M}+m^{2}\right]}(0)-\zeta_{\left[\mathcal{M}+m^{2}\right]}^{\prime}(0) .
\end{aligned}
$$

To compute the determinant ratio, we define the zeta function difference

$$
\zeta(s) \equiv \zeta_{\left[\mathcal{M}+m^{2}\right]}(s)-\zeta_{\left[\mathcal{M}^{\text {free }}+m^{2}\right]}(s) .
$$

Thus we need to compute the zeta function and its derivative, each evaluated at $s=0$. In general, the zeta function at $s=0$ is related to the heat kernel coefficient, $a_{d / 2}(\mathcal{P})$, associated with the operator $\mathcal{P}[6]: \zeta_{\mathcal{P}}(0)=a_{d / 2}(\mathcal{P})$. For the operator $\mathcal{P}=-\Delta-E$, these heat kernel coefficients are known [6], and we find the standard results

$$
\zeta(0)= \begin{cases}-\frac{1}{2} \int_{0}^{\infty} \mathrm{d} r r V(r), & d=2 \\ 0, & d=3 \\ \frac{1}{16} \int_{0}^{\infty} \mathrm{d} r r^{3} V\left(V+2 m^{2}\right), & d=4 .\end{cases}
$$

This gives the first term on the RHS of (12). Now we turn to the second term, the derivative of the zeta function at $s=0$, $\zeta^{\prime}(0)$. This can be evaluated using the relation to the familiar Jost functions of scattering theory [15]. Consider the radial eigenvalue equation

$$
\mathcal{M}_{(l) \phi_{(l), p}}=p^{2} \phi_{(l), p}
$$

where $\mathcal{M}_{(l)}$ is the Schrödinger-like radial operator defined in (3). A distinguished role is played by the so-called regular solution, $\phi_{(l), p}(r)$, which is defined to have the same behavior as $r \rightarrow 0$ as the solution without potential:

$$
\phi_{(l), p}(r) \underset{r \rightarrow 0}{\sim} \hat{j}_{l+\frac{(d-3)}{2}}(p r) .
$$

Here the spherical Bessel function is defined as

$$
\hat{j}_{l+\frac{(d-3)}{2}}(z)=\sqrt{\frac{\pi z}{2}} J_{l+\frac{d}{2}-1}(z) \text {. }
$$


The asymptotic behavior of the regular solution, $\phi_{(l), p}(r)$, as $r \rightarrow \infty$ defines the Jost function, $f_{l}(p)$, [15]

$\phi_{(l), p}(r) \underset{r \rightarrow \infty}{\sim} \frac{i}{2}\left[f_{l}(p) \hat{h}_{l+\frac{d-3}{2}}^{-}(p r)-f_{l}^{*}(p) \hat{h}_{l+\frac{d-3}{2}}^{+}(p r)\right]$.

Here $\hat{h}_{l+\frac{d-3}{2}}^{-}(p r)$ and $\hat{h}_{l+\frac{d-3}{2}}^{+}(p r)$ are the Riccati-Hankel functions

$$
\begin{aligned}
& \hat{h}_{l+\frac{d-3}{2}}^{+}(z)=i \sqrt{\frac{\pi z}{2}} H_{l+\frac{d}{2}-1}^{(1)}(z), \\
& \hat{h}_{l+\frac{d-3}{2}}^{-}(z)=-i \sqrt{\frac{\pi z}{2}} H_{l+\frac{d}{2}-1}^{(2)}(z) .
\end{aligned}
$$

As is well known from scattering theory [15], the analytic properties of the Jost function $f_{l}(p)$ strongly depend on the properties of the potential $V(r)$. Analyticity of the Jost function as a function of $p$ for $\mathfrak{\Im} p>0$ is guaranteed, if in addition to the aforementioned behavior as $r \rightarrow \infty$, we impose $V(r) \sim r^{-2+\varepsilon}$ for $r \rightarrow 0$, and continuity of $V(r)$ in $0<r<\infty$ (except perhaps at a finite number of finite discontinuities). For us, the analytic properties of the Jost function in the upper half plane will be of particular importance because they are related to the shifting of contours in the complex momentum plane.

By standard contour manipulations [6], the zeta function can be expressed in terms of the Jost functions as:

$\zeta(s)=\frac{\sin (\pi s)}{\pi} \sum_{l=0}^{\infty} \operatorname{deg}(l ; d) \int_{m}^{\infty} \mathrm{d} k\left[k^{2}-m^{2}\right]^{-s} \frac{\partial}{\partial k} \ln f_{l}(i k)$.

This representation is valid for $\Re s>d / 2$, and the technical problem is the construction of the analytic continuation of (19) to a neighborhood about $s=0$. If expression (19) were analytic at $s=0$, then we would deduce that

$$
\zeta^{\prime}(0)=-\sum_{l=0}^{\infty} \operatorname{deg}(l ; d) \ln f_{l}(\text { im }) .
$$

From the definition (17) of the Jost function,

$$
f_{l}(\mathrm{im})=\frac{\phi_{(l), i m}(\infty)}{\phi_{(l), i m}^{\text {free }}(\infty)}=\frac{\psi_{(l)}(\infty)}{\psi_{(l)}^{\text {free }}(\infty)},
$$

where $\psi_{(l)}(r)$ is defined in (9). Thus, the regulated expression (20) coincides with the formal partial wave expansion (5), using the Gelf'and-Yaglom result (2) for each $l$. However, the expansion (20) is divergent in positive integer dimensions. In the zeta function approach, the divergence of the formal sum in (20) is directly related to the need for analytic continuation of $\zeta(s)$ in $s$ to a region including $s=0$. From (19), this analytic continuation relies on the uniform asymptotic behavior of the Jost function $f_{l}(i k)$. Denoting this behavior by $f_{l}^{a s y m}(i k)$, the analytic continuation is achieved by adding and subtracting the leading asymptotic terms of the integrand in (19) to write

$$
\zeta(s)=\zeta_{f}(s)+\zeta_{a s}(s),
$$

where

$$
\begin{aligned}
& \zeta_{f}(s)=\frac{\sin (\pi s)}{\pi} \\
& \sum_{l=0}^{\infty} \operatorname{deg}(l ; d) \int_{m}^{\infty} \mathrm{d} k\left[k^{2}-m^{2}\right]^{-s} \frac{\partial}{\partial k}\left[\ln f_{l}(i k)-\ln f_{l}^{a s y m}(i k)\right],
\end{aligned}
$$

and

$$
\begin{aligned}
& \zeta_{a s}(s)=\frac{\sin (\pi s)}{\pi} \\
& \cdot \sum_{l=0}^{\infty} \operatorname{deg}(l ; d) \int_{m}^{\infty} \mathrm{d} k\left[k^{2}-m^{2}\right]^{-s} \frac{\partial}{\partial k} \ln f_{l}^{a s y m}(i k) .
\end{aligned}
$$

Ultimately we are interested in the analytic continuation of $\zeta(s)$ to $s=0$. As many asymptotic terms will be included in $f_{l}^{a s y m}(i k)$ as are necessary to make $\zeta_{f}(s)$ as given in (23) analytic around $s=0$. On the other hand, for $\zeta_{a s}(s)$ the analytic continuation to $s=0$ can be constructed in closed form using an explicit representation of the asymptotic behavior of the Jost function, derived in the next section.

\subsection{Asymptotics of the Jost function}

The asymptotics of the Jost function $f_{l}(i k)$ follows from standard results in scattering theory [15]. In particular, the partial-wave Lippmann-Schwinger integral equation for the regular solution

$\phi_{(l), i k}(r)=I_{v}(k r)$

$+\int_{0}^{r} \mathrm{~d} r^{\prime} r^{\prime}\left[I_{v}(k r) K_{v}\left(k r^{\prime}\right)-I_{v}\left(k r^{\prime}\right) K_{v}(k r)\right] V\left(r^{\prime}\right) \phi_{(l), i k}\left(r^{\prime}\right)$.

leads to an iterative expansion for $f_{l}(i k)$ in powers of the potential $V(r)$. For dimensions $d \leq 4$, we need at most the $\mathcal{O}(V)$ and $\mathcal{O}\left(V^{2}\right)$ terms of $\ln f_{l}(i k)$ :

$$
\begin{aligned}
\ln f_{l}(i k) & =\int_{0}^{\infty} \mathrm{d} r r V(r) K_{v}(k r) I_{v}(k r) \\
& -\int_{0}^{\infty} \mathrm{d} r r V(r) K_{v}^{2}(k r) \int_{0}^{r} \mathrm{~d} r^{\prime} r^{\prime} V\left(r^{\prime}\right) I_{v}^{2}\left(k r^{\prime}\right)+\mathcal{O}\left(V^{3}\right) .
\end{aligned}
$$

This iterative scheme effectively reduces the calculation of the asymptotics of the Jost function to the well-known uniform asymptotics of the modified Bessel functions $K_{v}$ and $I_{v}$. Using these asymptotics, wedefine $\ln f_{l}^{a s y m}(i k)$ as the $\mathcal{O}(V)$ and $\mathcal{O}\left(V^{2}\right)$ parts of this uniform asymptotic expansion:

$\ln f_{l}^{\text {asym }}(i k) \equiv \frac{1}{2 v} \int_{0}^{\infty} \mathrm{d} r \frac{r V(r)}{\left[1+\left(\frac{k r}{v}\right)^{2}\right]^{\frac{1}{2}}}+\frac{1}{16 v^{3}} \int_{0}^{\infty} \mathrm{d} r \frac{r V(r)}{\left[1+\left(\frac{k r}{v}\right)^{2}\right]^{\frac{3}{2}}}\left[1-\frac{6}{\left[1+\left(\frac{k r}{v}\right)^{2}\right]}+\frac{5}{\left[1+\left(\frac{k r}{v}\right)^{2}\right]^{2}}\right]-\frac{1}{8 v^{3}} \int_{0}^{\infty} \mathrm{d} r \frac{r^{3} V^{2}(r)}{\left[1+\left(\frac{k r}{v}\right)^{2}\right]^{\frac{3}{2}}}$. 


\subsection{Computing $\zeta_{f}^{\prime}(\mathbf{0})$}

By construction, $\zeta(s)$, defined in (23), is now well defined at $s=0$, and we find

$$
\xi_{f}^{\prime}(0)=-\sum_{l=0}^{\infty} \operatorname{deg}(l ; d)\left[\ln f_{l}(i m)-\ln f_{l}^{a s y m}(i m)\right] \text {. }
$$

This form is suitable for straightforward numerical computation, as the Jost function $f_{l}(\mathrm{im})$ can be computed using (9) and (21), while $\ln f_{l}^{a s y m}(\mathrm{im})$ can be computed using (27). With the subtraction of $\ln f_{l}^{a s y m}(\mathrm{im})$ in (28), the $l$ sum is now convergent.

However, it is possible to find an even simpler expression. It turns out that the subtraction in (28) is an over-subtraction. To see this, expand $\ln f_{l}^{a s y m}(\mathrm{im})$ into its large $l$ behavior as follows:

$$
\begin{aligned}
& \ln f_{l}^{a s y m}(i m) \sim \frac{1}{2 v} \int_{0}^{\infty} \mathrm{d} r r V(r)-\frac{1}{8 v^{3}} \int_{0}^{\infty} \mathrm{d} r r^{3} V\left(V+2 m^{2}\right)+\frac{1}{2 v} \int_{0}^{\infty} \mathrm{d} r r V(r)\left\{\left[1+\left(\frac{m r}{v}\right)^{2}\right]^{-\frac{1}{2}}-1+\frac{1}{2}\left(\frac{m r}{v}\right)^{2}\right\} \\
& +\frac{1}{16 v^{3}} \int_{0}^{\infty} \mathrm{d} r \frac{r V(r)}{\left[1+\left(\frac{m r}{v}\right)^{2}\right]^{2}}\left[1-\frac{6}{\left[1+\left(\frac{m r}{v}\right)^{2}\right]}+\frac{5}{\left[1+\left(\frac{m r}{v}\right)^{2}\right]^{2}}\right]-\frac{1}{8 v^{3}} \int_{0}^{\infty} \mathrm{d} r r^{3} V^{2}(r)\left\{\left[1+\left(\frac{m r}{v}\right)^{2}\right]^{-\frac{3}{2}}-1\right\}
\end{aligned}
$$

The first term is $\mathcal{O}\left(\frac{1}{l}\right)$, and the second is $\mathcal{O}\left(\frac{1}{l^{3}}\right)$, while the remaining terms are all $\mathcal{O}\left(\frac{1}{l^{5}}\right)$. In dimensions $d \leq 4$, the degeneracy factor $\operatorname{deg}(l ; d)$ is at most quadratic in $l$, and so these last terms are finite when summed over $l$ in (28). (In fact, in $d=2$ and $d=3$, the $\mathcal{O}\left(\frac{1}{l^{3}}\right)$ terms are also finite when summed over $l$.) In the next section we show that these finite terms cancel exactly against corresponding terms arising in the evaluation of $\zeta_{a s}^{\prime}(0)$. Thus, for $\zeta^{\prime}(0)=\zeta_{f}^{\prime}(0)+\zeta_{a s}^{\prime}(0)$, we only actually need to subtract the leading large $l$ terms in (29), rather than the full asymptotics in (27).

\subsection{Computing $\zeta_{a s}^{\prime}(0)$}

The explicit form of the asymptotic terms in (28) provides the analytic continuation to $s=0$ of $\zeta_{a s}(s)$, as defined in (24). The $k$ integrals are done using

$$
\int_{m}^{\infty} \mathrm{d} k\left[k^{2}-m^{2}\right]^{-s} \frac{\partial}{\partial k}\left[1+\left(\frac{k r}{v}\right)^{2}\right]^{-\frac{n}{2}}=-\frac{\Gamma\left(s+\frac{n}{2}\right) \Gamma(1-s)}{\Gamma\left(\frac{n}{2}\right)} \frac{\left(\frac{v}{m r}\right)^{n} m^{-2 s}}{\left[1+\left(\frac{v}{m r}\right)^{2}\right]^{s+\frac{n}{2}}} .
$$

Therefore, we find

$$
\begin{aligned}
& \zeta_{a s}(s)=-\sum_{l=0}^{\infty} \operatorname{deg}(l ; d)\left[\int _ { 0 } ^ { \infty } \mathrm { d } r r ^ { 1 + 2 s } V ( r ) \left\{\frac{1}{2} \frac{\Gamma\left(s+\frac{1}{2}\right)}{\Gamma(s) \Gamma\left(\frac{1}{2}\right)} \frac{v^{-1-2 s}}{\left[1+\left(\frac{m r}{v}\right)^{2}\right]^{s+1 / 2}}+\frac{1}{16} \frac{\Gamma\left(s+\frac{3}{2}\right)}{\Gamma(s) \Gamma\left(\frac{3}{2}\right)} \frac{v^{-3-2 s}}{\left[1+\left(\frac{m r}{v}\right)^{2}\right]^{s+3 / 2}}\right.\right. \\
& \left.-\frac{3}{8} \frac{\Gamma\left(s+\frac{5}{2}\right)}{\Gamma(s) \Gamma\left(\frac{5}{2}\right)} \frac{v^{-3-2 s}}{\left[1+\left(\frac{m r}{v}\right)^{2}\right]^{s+5 / 2}}+\frac{5}{16} \frac{\Gamma\left(s+\frac{7}{2}\right)}{\Gamma(s) \Gamma\left(\frac{7}{2}\right)} \frac{v^{-3-2 s}}{\left[1+\left(\frac{m r}{v}\right)^{2}\right]^{s+7 / 2}}\right\} \\
& \left.-\int_{0}^{\infty} \mathrm{d} r r^{3+2 s} V^{2}(r) \frac{1}{8} \frac{\Gamma\left(s+\frac{3}{2}\right)}{\Gamma(s) \Gamma\left(\frac{3}{2}\right)} \frac{v^{-3-2 s}}{\left[1+\left(\frac{m r}{v}\right)^{2}\right]^{s+3 / 2}}\right] .
\end{aligned}
$$

We now subtract sufficiently many terms inside thesum to ensure the analytic continuation of $\xi_{a s}(s)$ to $s=0$. The added back terms produce Riemann zeta function terms, such as $\xi_{R}(2 s+1)$, whose analytic continuation is immediate. For example, in $d=4$, we find 


$$
\begin{aligned}
& \left.\zeta_{a s}^{\prime}(0)\right|_{d=4}=\frac{1}{8} \int_{0}^{\infty} \mathrm{d} r r^{3} V\left(V+2 m^{2}\right)\left[\ln \left(\frac{r}{2}\right)+\gamma+1\right]-\int_{0}^{\infty} \mathrm{d} r r V(r)\left\{\frac{1}{2} \sum_{v=1}^{\infty} v\left[\left(1+\left(\frac{m r}{v}\right)^{2}\right)^{-\frac{1}{2}}-1+\frac{1}{2}\left(\frac{m r}{v}\right)^{2}\right]\right. \\
& \left.+\frac{1}{16} \sum_{v=1}^{\infty} \frac{1}{v}\left[\left(1+\left(\frac{m r}{v}\right)^{2}\right)^{-\frac{3}{2}}-6\left(1+\left(\frac{m r}{v}\right)^{2}\right)^{-\frac{5}{2}}+5\left(1+\left(\frac{m r}{v}\right)^{2}\right)^{-\frac{7}{2}}\right]\right\}+\frac{1}{8} \int_{0}^{\infty} \mathrm{d} r r^{3} V^{2}(r) \sum_{v=1}^{\infty} \frac{1}{v}\left[\left(1+\left(\frac{m r}{v}\right)^{2}\right)^{-\frac{3}{2}}-1\right] .
\end{aligned}
$$

Notice that the terms involving summation over $v$ cancel exactly against identical terms in $\zeta_{f}^{\prime}(0)$ from (29), after those terms are summed over $l$ with the $d=4$ degeneracy factor $v^{2}=(l+1)^{2}$. Furthermore, note that the $\ln r$ term inside the integral on the second line of (32) is precisely of the same form as the renormalization term in (14), so the $\ln \mu$ in (12) combines with $\ln r$ to form the dimensionless combination $\ln (\mu r)$ in (8).

The analogous computations for $d=2$ and $d=3$ lead to (6) and (7), respectively [14].

\section{Conclusions and applications}

The mathematical results reported here are the formulas in (6)-(8), which provide simple new expressions for the determinant of a radially separable partial differential operator of the form $-\Delta+m^{2}+V(r)$. This generalizes the Gel'fand-Yaglom result (2) to higher dimensions, and greatly increases the class of differential operators for which the determinant can be computed simply and efficiently. The derivation presented here [14] uses the zeta function definition of the determinant, but the same expressions can be found using the radial WKB approach of [12, 13]. Furthermore, it can be shown [14] how these expressions relate to the Feynman diagrammatic definition of the determinant based on dimensional regularization [16]. These superficially different expressions are in fact equal, although the zeta function expression is considerably simpler to implement.

These results lead to many direct applications in quantum field theory, where they extend the class of solvable fluctuation determinant problems away from the restrictive class of constant background fields, or one dimensional background fields, to the more general class of separable higher dimensional background fields. Mathematically, this represents a small but surprisingly non-trivial step towards more general partial differential operators. While this is still a small class of partial differential operators, it is large enough to have many important applications in quantum field theory, for example the study of quantum fluctuations in the presence of vortices, monopoles, sphalerons, instantons, domain walls (branes), etc ...

A number of generalizations could be made. First, in certain quantum field theory applications the determinant may have zero modes, and correspondingly one is actually interested in computing the determinant with these zero modes removed. Our method provides a simple way to compute such determinants [12]. A systematic study of this approach, exploiting the relation between zero modes and topology, would be extremely interesting. Another important generalization is to include directly the matrix structure that arises from
Dirac-like differential operators and from non-abelian gauge degrees of freedom. The Feynman diagrammatic approach is well developed for such separable problems [17]; for example it has been applied to the fluctuations about the electroweak sphaleron $[18,19]$ and to compute the metastability of the electroweak vacuum [20]. More recently, the angular momentum cut-off method has been used to compute the full mass dependence of the fermion determinant in a four dimensional Yang-Mills instanton background [13], to compute the fermion determinant in a background instanton in the two dimensional chiral Higgs model [21], and to address the fluctuation problem for false vacuum decay in curved space [22]. A unified zeta function analysis should be possible, as there is a straightforward generalization of the Gel'fand-Yaglom result (2) to systems of ordinary differential operators [10].

Finally, to conclude, the real mathematical challenge is to ask if the restriction of separability can be loosened. This is a difficult problem, but it is clear that any progress will be interesting.

\section{Acknowledgments}

I sincerely thank the organizer, Miloslav Znojil, and also Pavel Exner, for the kind invitation to Villa Lana and the beautiful city of Prague. I acknowledge support from the DFG through the Mercator Guest Professor Program, and from the US DOE through grant DE-FG02-92ER40716. This talk is based on work done in collaboration with Klaus Kirsten.

\section{References}

[1] Ray, D. B., Singer, I. M.: R-Torsion and the Laplacian on Riemannian Manifolds, Adv. Math. Vol. 7 (1971), p. 145-210.

[2] Hawking, S. W.: Zeta Function Regularization of Path Integrals in Curved Space-time, Commun. Math. Phys. Vol. 55 (1977), p. 133-148.

[3] D'Hoker E., Phong, D. H.: On Determinants of Laplacians on Riemann Surfaces, Commun. Math. Phys. Vol. 104 (1986), p. 537-545.

[4] Sarnak, P.: Determinants of Laplacians, Commun. Math. Phys. Vol. 110 (1987), p. 113-120.

[5] Elizalde, E., Odintsov, S. D., Romeo, A., Bytsenko, A. A., Zerbini, S.: Zeta Regularization Techniques with Applications, World Scientific, Singapore, 1994.

[6] Kirsten, K.: Spectral Functions in Mathematics and Physics, Chapman-Hall, Boca Raton, 2002.

[7] Gelfand. I. M., Yaglom, A. M.: Integration in Functional Spaces and its Applications in Quantum Physics, J. Math. Phys. Vol. 1 (1960), p. 48-69. 
[8] Levit, S., Smilansky, U.: A Theorem on Infinite Products of Eigenvalues of Sturm-Liouville Type Operators, Proc. Am. Math. Soc. Vol. 65 (1977), p. 299-303.

[9] Forman, R.: Functional Determinants and Geometry, Invent. Math. Vol. 88 (1987), p. 447-493; Erratum, ibid 108 (1992), p. 453-454.

[10] Kirsten, K., McKane, A. J.: Functional Determinants by Contour Integration Methods, Annals Phys. Vol. 308 (2003), p. 502-527 [arXiv:math-ph/0305010]; Functional Determinants for General Sturm-Liouville Problems, J. Phys. A Vol. 37 (2004), p. 4649-4670 [arXiv:math-ph/0403050].

[11] Kleinert, H.: Path Integrals in Quantum Mechanics, Statistics, Polymer Physics, and Financial Markets, World Scientific, Singapore, 2004.

[12] Dunne, G. V., Min, H.: Beyond the Thin-Wall Approximation: Precise Numerical Computation of prefactors in False Vacuum Decay, Phys. Rev. D Vol. 72 (2005), 125004 [arXiv:hep-th/0511156].

[13] Dunne, G. V., Hur, J., Lee, C., Min, H.: Instanton Determinant with Arbitrary Quark Mass: WKB Phase-Shift Method and Derivative Expansion, Phys. Lett. B Vol. 600 (2004), p. 302-313 [arXiv:hep-th/0407222];

Precise Quark Mass Dependence of instanton Determinant, Phys. Rev. Lett. Vol. 94 (2005), 072001

[arXiv:hep-th/0410190];

Calculation of QCD Instanton Determinant with Arbitrary Mass, Phys. Rev. D Vol. 71 (2005), 085019 [arXiv:hep-th/0502087].

[14] Dunne, G. V., Kirsten, K.: Functional Determinants for Radial Operators, J. Phys. A Vol. 39 (2006), p. 11915-11928 [arXiv:hep-th/0607066].

[15] Taylor, J. R.: Scattering Theory, Wiley, New York, 1972.

[16] Baacke, J., Lavrelashvili, G.: One-Loop Corrections to the Metastable Vacuum Decay, Phys. Rev. D Vol. 69 (2004), 025009 [arXiv:hep-th/0307202].

[17] Baacke, J.: Numerical Evaluation of the One Loop Effective Action in Static Backgrounds with Spherical Symme- try, Z. Phys. C Vol. 47 (1990), p. 263-268; The Effective Action of a Spin 1/2 Field in the Background of a Chiral Soliton, Z. Phys. C Vol. 53 (1992), p. 407-411.

[18] Carson, L., Li, X., McLerran, L. D., Wang, R. T.: Exact Computation of the Small Fluctuation Determinant Around a Sphaleron, Phys. Rev. D Vol. 42, (1990), p. 2127-2143

[19] Baacke, J., Junker, S.: Quantum Fluctuations Around the Electroweak Sphaleron, Phys. Rev. D Vol. 49 (1994), p. 2055-2073 [arXiv:hep-ph/9308310]; Quantum Fluctuations of the Electroweak Sphaleron: Erratum and addendum, Phys. Rev. D Vol. 50 (1994), p. 4227-4228 [arXiv:hep-th/9402078].

[20]Isidori, G. Ridolfi, G., Strumia, A.: On the Metastability of the Standard Model Vacuum, Nucl. Phys. B Vol. 609 (2001), p. 387-409 [arXiv:hep-ph/0104016].

[21] Burnier, Y., Shaposhnikov, M.: One-Loop Fermionic Corrections to the Instanton Transition in Two Dimensional Chiral Higgs Model, Phys. Rev. D Vol. 72 (2005), 065011 [arXiv:hep-ph/0507130].

[22] Dunne, G. V., Wang, Q. H.: Fluctuations about Cosmological Instantons, Phys. Rev. D Vol. 74 (2006), 024018 [arXiv:hep-th/0605176].

Prof. Gerald V. Dunne

phone: +1 8604864978

e-mail: dunne@phys.uconn.edu

Department of Physics

University of Connecticut

Storrs, CT 06269, USA

(and

Institut fürTheoretische Physik

Universität Heidelberg

Philosophenweg 16

69120 Heidelberg, Germany) 